\author{
S. Schmidt \\ P. Chevallier \\ S. Novellas \\ E. Gelsi \\ G. Vanbiervliet \\ A. Tran \\ P. Schnyder \\ J. N. Bruneton
}

\section{Choledocholithiasis: repetitive thick-slab single-shot projection magnetic resonance cholangiopancreaticography versus endoscopic ultrasonography}

Received: 6 January 2006

Revised: 6 June 2006

Accepted: 23 June 2006

Published online: 29 August 2006

(C) Springer-Verlag 2006
S. Schmidt · P. Schnyder

Department of Diagnostic and

Interventional Radiology, CHUV,

Rue du Bugnon, 1011,

Lausanne, Switzerland

P. Chevallier - S. Novellas ·

J. N. Bruneton

Service d'Imagerie Médicale,

Hôpital Archet II, CHU,

Nice, France

E. Gelsi · G. Vanbiervliet · A. Tran

Service de Gastroenterologie,

Hôpital Archet II, CHU,

Nice, France

S. Schmidt $(\bowtie)$

Service de radiodiagnostic et radiologie

interventionnelle, Centre Hospitalier

Universitaire Vaudois-CHUV,

Rue du Bugnon 1011,

Lausanne, Switzerland

e-mail: sabine.schmidt@chuv.ch

Tel.: +41-21-3144607

Fax: +41-21-3144443

\author{
Abstract This prospective study \\ compares repetitive thick-slab single- \\ shot projection magnetic resonance \\ cholangiopancreatography (MRCP) \\ with endoscopic ultrasonography \\ (EUS) for the detection of chole- \\ docholithiasis. Fifty-seven consecu- \\ tive patients (36 women, mean age 61) \\ referred for suspected choledocho- \\ lithiasis underwent MRCP, followed \\ by EUS. Each procedure was per- \\ formed by different operators blinded \\ to the results of the other investiga- \\ tion. MR technique included a turbo \\ spin-echo T2-weighted axial sequence \\ with selective fat saturation (SPIR/ \\ TSE, TE=70 ms, TR=1,600 ms), fol- \\ lowed by coronal dynamic MRCP. \\ The same thick-slab slice was se- \\ quentially acquired 12 times as breath- \\ hold single-shot projection imaging \\ (SSh, TE $=900 \mathrm{~ms}, \mathrm{TE}=8,000 \mathrm{~ms}$ ) \\ centred on the common bile duct \\ (CBD). Two experienced radiologists \\ independently and blindly evaluated \\ MR images for the detection of CBD \\ stones. Their inter-observer agreement \\ kappa was determined. Secondly, the \\ two observers read MR images in \\ consensus again. CBD stones were
}

demonstrated in 18 out of 57 patients $(31.6 \%)$ and confirmed by endoscopic retrograde cholangiography (ERCP, $n=17$ ) or intraoperative cholangiography $(n=1)$. Clinical follow-up served as the "gold standard" in patients with negative results without following invasive procedure $(n=28)$. Sensitivity, specificity, accuracy, positive and negative predictive value for MRCP resulting from consensus reading were $94.9 \%, 94.4 \%, 94.7 \%$, $97.4 \%$ and $89.5 \%$, respectively. Corresponding values of EUS were $97.4 \%, 94.4 \%, 96.5 \%, 97.4 \%$ and $94.4 \%$. Inter-observer agreement kappa was 0.81 . Repetitive thick-slab single-shot projection MRCP is an accurate non-invasive imaging modality for suspected choledocholithiasis and should be increasingly used to select those patients who require a subsequent therapeutic procedure, namely ERCP.

Keywords Magnetic resonance cholangiopancreaticography · Endoscopic ultrasonography · Choledocholithiasis .

Common bile duct $\cdot$ Calculi

\section{Introduction}

Choledocholithiasis occurs in $15-20 \%$ of patients with gall bladder stones. Recurrent or persistent common bile duct (CBD) stones are detected in $1-5 \%$ of patients after cholecystectomy performed for calculi [1].
Endoscopic retrograde cholangiopancreaticography (ERCP) continues to be considered as the standard of reference for the detection of bile duct stones with the possibility of simultaneous treatment. Nevertheless, ERCP remains an invasive investigation and it is greatly operator dependent. Associated complications, such as secondary 
pancreatitis, occur in $1-5 \%$ of cases [2-4]. Unnecessary, purely diagnostic ERCP should therefore be avoided [3].

Many authors consider endoscopic ultrasonography (EUS) as the most reliable pretherapeutic diagnostic tool for choledocholithiasis, superior to magnetic resonance cholangiopancreaticography (MRCP) and as accurate as ERCP [5-9]. EUS is less invasive than ERCP, but, similar to ERCP, the operator must be skilled and experienced. Therefore, EUS is not readily available and diagnostic results are highly operator-dependent $[5,7,10,11]$. The overall diagnostic value of EUS for choledocholithiasis has been reported as a sensitivity of $80-100 \%$, specificity of $88-100 \%$ and diagnostic accuracy of $95-98 \%$ [5, 6, 8-14]. MRCP has nowadays become a commonly used and easily reproductive imaging modality. The acquisition of cholangiographic sequences can be performed on most middle- or high-field MR scanners without special training or intravenous injection of contrast medium, thus rendering MRCP a completely non-invasive technique that is little influenced by the operator. The overall diagnostic value of MRCP has been proven to be similar to that of ERCP [15] and EUS for the detection of choledocholithiasis, provided that CBD dilatation was associated in the majority of included patients. The sensitivity varies between 80 and $100 \%$, specificity between 85 and $100 \%$, and a diagnostic accuracy of 94-97\% [6, 9, 10, 12, 13, 16-25].

However, if one takes into account only non-obstructive CBD stones, MRCP is less sensitive. Statistical results for this subgroup are also quite variable in the literature $[9,10$, $18,23]$. This variability reflects differences in equipment, the chosen MR technique and the experience of the radiologists in image interpretation. Only the repetition of thick-slab single-shot projection MRCP enables the visualisation of the entire length of the CBD, which seems mandatory for the reliable detection or exclusion of choledocholithiasis.

Diagnostic results of EUS have been shown to be independent of stone size or CBD dilatation [5]. Our objective was, therefore, the prospective and direct comparison of MRCP with EUS for the detection of choledocholithiasis, relying on dynamic and sequential thick-slab MR imaging (MRI) in order to visualise the entire length of the CBD, in particular the sphincter complex.

\section{Patients and methods}

\section{Patients}

From June 2003 through October 2004, 61 consecutive adult patients with suspected choledocholithiasis were considered to be included in this monocentric and prospective evaluation comparing MRI versus EUS, performed within a delay of 5 days. From the initial 61 patients we had to exclude two with biliodigestive anastomosis (choledochojejunostomy, Billroth II), rendering EUS and ERCP impossible. Two other patients were also excluded because of inadequate clinical follow-up.

Our final study population was composed of 57 patients [36 women, mean age 61 years $( \pm 17.3)$, range $=27$ 92 years], among them 15 with a history of previous cholecystectomy for calculi. Their clinical data are resumed in Table 1.

Permission of the institutional ethical committee had been obtained for this study, as well as informed oral consent from the patients.

\section{Technique of MRI}

MRI was performed with a 1-Tesla magnet (Intera, Philips Medical Systems, Best, The Netherlands) using a torso phased-array receiving coil placed around the upper abdomen. Patients were examined in the supine position without any previous preparation, in particular no premedication and neither was an oral contrast medium for suppression of possible hyperintense gastric or duodenal content administered [26].

A survey MR examination was followed by a turbo spinecho T2-weighted axial MR sequence with selective fat saturation and respiratory compensation, centred on the hepatobiliary region (SPIR/TSE) using the following parameters: repetition time (TR) 1,600 m, echo time (TE) $70 \mathrm{~ms}$, turbo factor 24, number of excitations (NEX) 2, slice thickness $7 \mathrm{~mm}$, matrix size $256 \times 256$ and field of view (FOV) $435 \times 435 \mathrm{~mm}$. Twenty-five images were obtained within about $38 \mathrm{~s}$ (factor SENSE 1). Saturation bands were prescribed above and below the region or interest. Axial images served as guide for choosing the angle of coronal sections to be obtained at MR cholangiography.

Table 1 Clinical data of the study population

\begin{tabular}{ll}
\hline Clinical data & $\begin{array}{l}\text { Number } \\
\text { of patients }\end{array}$ \\
\hline
\end{tabular}

Pain situated in the upper right abdominal quadrant 29

or epigastric region and fever or jaundice with

associated increase of serum alkaline phosphatase

(normal 39-105 IU/1), serum gamma-glutamyl

transpeptidase (normal 5-36 IU/l) or total bilirubin

(normal 3-22 IU/1) possibly in combination with an inflammatory syndrome (C-reactive protein $>5 \mathrm{IU}$ )

Acute pancreatitis of suspected biliary origin $\quad 21$

Biological cholestasis with acute fever or jaundice 5

Acute upper abdominal pain and a clinical history 2

suggestive of biliary stones disease, such is known cholecystolithiasis or acutely dilated CBD seen on

abdominal US or CT after cholecystectomy

Total study population 
MRCP included a breath-hold, turbo spin-echo sequence (single shot) using a TR $8,000 \mathrm{~ms}$, TE $900 \mathrm{~ms}$, turbo factor 205 , NEX 1 and a matrix size $256 \times 205$ reconstructed in $512 \times 512$ pixels for a FOV of $250 \times 340 \mathrm{~mm}$. Single shot repetitive projection imaging comprised 12 dynamic slices of 40-mm thickness, in the right anterior oblique coronal plane and centred on the CBD, especially on the Vaterian sphincter complex. Each section was acquired within a single breath-hold of $2 \mathrm{~s}$. Saturation bands over patient's left and right flank were used.

Film reading of MR images was done by two boardcertified radiologists with extensive practical experience in hepatobiliary MRI. They were both blinded to the clinical probability of choledocholithiasis as well as to the final outcome. These two radiologists read the same images again in consensus, still without any clinical information.

MRI was considered normal whenever the maximal diameter of the CBD at the level of the hepatic hilus measured $<8 \mathrm{~mm}$, respectively $<11 \mathrm{~mm}$ in patients older than 70 years or after cholecystectomy. Furthermore, the entire course of the CBD, including the sphincter complex, had to be clearly visible and characterised by a homogeneous hypersignal on either of the two T2-weighted MR sequences. The two radiologists also evaluated in consensus the number of repetitions necessary to visualise the lumen of the sphincter complex for the first time, and how often it could be seen in a relaxed state during the 12 repetitive MRCP sequences performed.

MRI was considered positive whenever a round, oval or multifaceted area of signal void was seen within the T2 hyperintense lumen of the CBD. It must be detected on either of the two different MR sequences (axial or coronal oblique plane) performed; its size was exactly recorded. Whenever several CBD stones were depicted in the same patient, the largest one of them was statistically taken into account.

In case of biliary sludge, seen as hypointense sediment accumulating within the distal CBD, MRI was also considered positive.

\section{Technique of EUS}

In all patients MRI preceded EUS, which had to follow within 5 days.

Each EUS was performed and interpreted by a staff gastroenterologist known for broad experience in endosonography, using the system PENTAX EG-3630UR with a radial technology ultrasound probe functioning at 510 Mhz. It was connected to an HITACHI Alpha Harmonic ultrasound machine of the newest generation (2004). Patients were investigated under general anaesthesia (introduced by propofol) in the left lateral decubitus position after fasting for at least $8 \mathrm{~h}$. After duodenal intubation, the transducer was advanced to the third part of duodenum. The progressive withdrawal of the echoendo- scope towards the duodenal bulb allowed the visualisation of the Vaterian sphincter complex and the distal CBD first, followed by the proximal CBD, the hepatic and intrahepatic bile ducts. The examination took about $15 \mathrm{~min}$.

As to the normal size of the CBD, the same criteria as described above for MRI were applied.

CBD stones were diagnosed with EUS whenever a hyperechoic focus with or without posterior acoustic shadowing was detected within the lumen of the CBD [7].

\section{Proof of disease}

MRI and EUS were performed by different operators blinded to the result of the other method used. In case of negative EUS, the corresponding MRCP result was immediately communicated as soon as EUS was finished, while the patient was being kept on the table of the endoscopy unit, still under general anaesthesia. If any or both imaging modalities detected a CBD stone or any unexplained common bile duct dilatation, immediate ERCP or cholangiography during cholecystectomy was performed and considered as the "gold standard".

In case of both negative EUS and MRCP, and no following invasive procedure, the clinical follow-up served as the gold standard.

\section{Statistical analysis}

Image quality was assessed for each of the two MR sequences and graded according to the depiction of the intra-and extrahepatic bile ducts, and the papilla. We used the scores 1 (excellent=visualisation of all these three anatomical structures), 2 (good=identification of two out of three anatomical structures) and 3 (moderate $=$ detection of one out of three anatomical structures).

Using the above-defined gold standard, sensitivity, specificity, accuracy, positive (PPV) and negative predictive value (NPV) of MCRP and EUS for choledocholithiasis were calculated $(95 \%$ confidence interval, $P<0.05)$, whereby MRCP results resulted from radiologists' consensus reading.

Inter-observer agreement concerning the detection of CBD stones was determined between the two radiologists, according to the common kappa rating defined by Fleiss [27]: kappa $<0.4=$ poor, $0.4-0.75=$ good, and $>0.75=$ excellent agreement.

Presence of CBD dilatation seen on MRI and EUS was compared (kappa). Linear regression was applied for correlating the degree of CBD dilatation and correlating the stone size detected by MRI and EUS using the Pearson's correlation coefficient $(P<0.05)$.

Altogether, the chi-square statistic and Fisher exact test were used to compare categorical variables, and the 
Student's $t$-test for evaluation of continuous variables, when appropriate.

\section{Results}

The mean interval time between MRI and EUS was 1.9 days (range $0-5$ days).

EUS was successfully performed in each of the 57 patients without any complication.

Altogether, MRI could be interpreted in all patients. The technical quality of the axial T2-weighted sequences was graded excellent in 40 patients, good in 16 and moderate in one patient. Dynamic MRCP images were considered excellent in 26 patients, good in 29 and moderate in two patients.

Common bile duct stones were detected in 18 out of 57 patients (31.6\%) (Figs. 1, 2, 3), confirmed by ERCP in 17 patients and by intraoperative cholangiography in one patient. The delay between EUS and ERCP/intraoperative cholangiography was 4.1 days at an average (range 0 9 days).

The mean clinical follow-up of the 28 patients with negative result for both techniques, EUS and MRCP, and

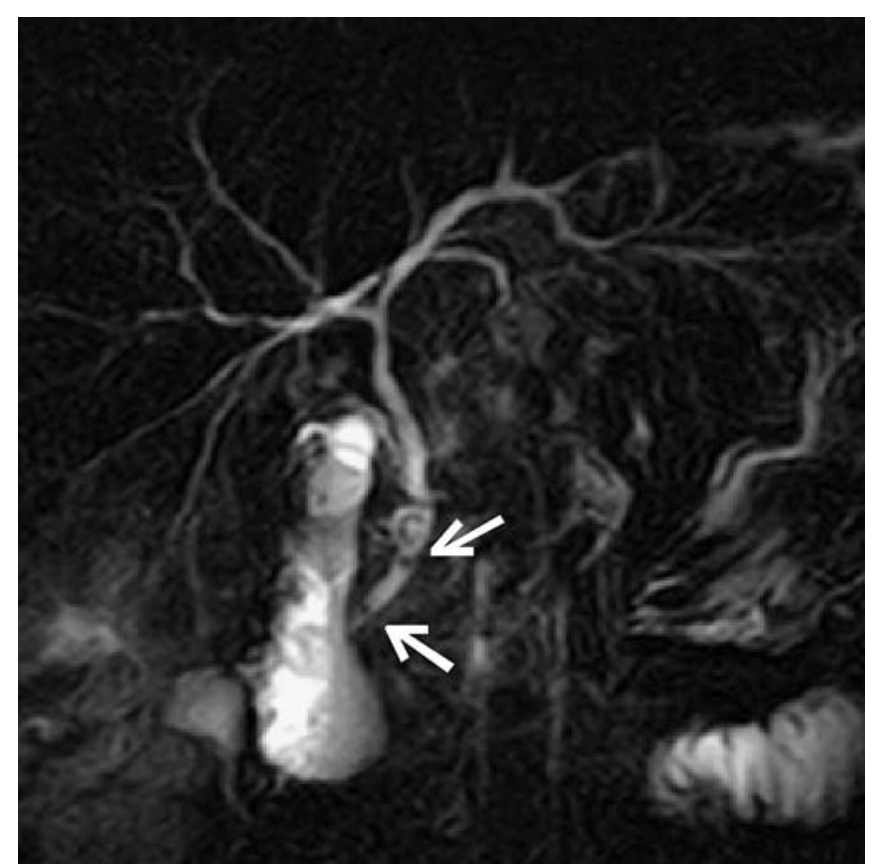

Fig. 1 A 30-year-old woman with biological cholestasis and acute pain of the right hypochondrium. Prior abdominal sonography had already detected several small gallbladder stones, but no relevant bile duct dilatation. Coronal oblique T2-weighted thick-slab singleshot turbo spin-echo MRCP sequence confirms microlithiasis of the gall bladder (not well demonstrated on this image) and shows two signal voids measuring $2 \mathrm{~mm}$ each (arrows) situated in the distal portion of CBD causing slight dilatation of the intra-and extrahepatic bile ducts. EUS performed $24 \mathrm{~h}$ later confirmed these microlithiasis, as well as following ERCP with calculi extraction and sphincterotomy

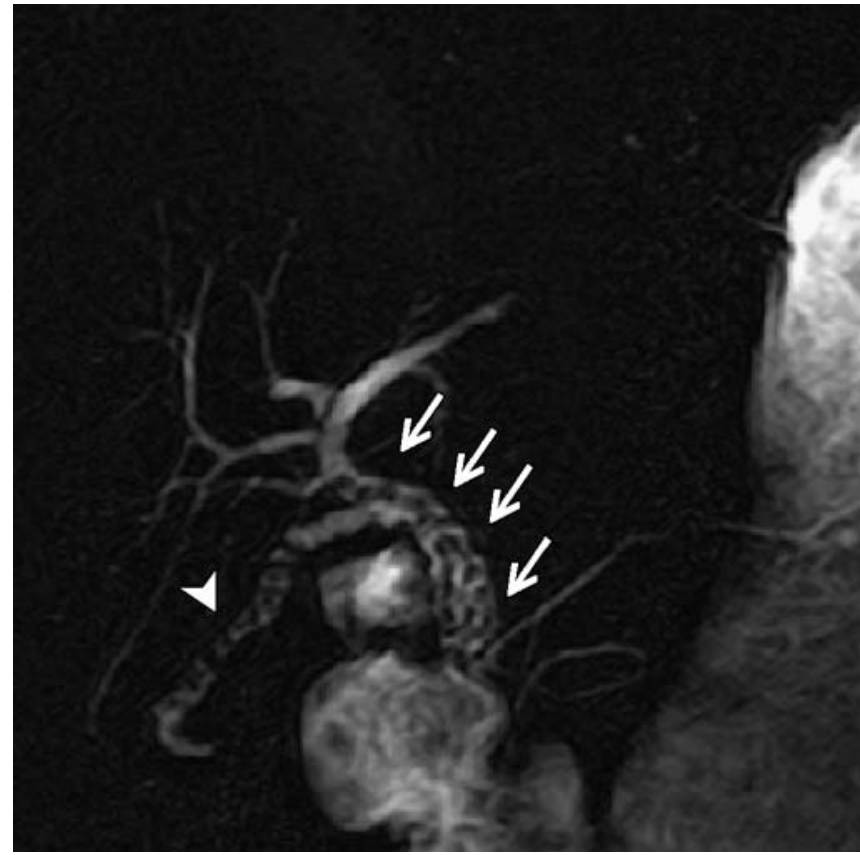

Fig. 2 A 25-year-old man with acute epigastric pain, known for chronic calculous cholecystitis. Coronal oblique T2-weighted thickslab single-shot turbo spin-echo MRCP sequence reveals numerous macrolithiasis obstructing the whole CBD (arrows) with intrahepatic bile duct dilatation, later confirmed by EUS and ERCP. Note also the multiple calculi situated in the long cystic duct (arrowhead) causing obstruction of the gall bladder which is sclerosed, contracted and atrophied, therefore not visualised on this image

without following invasive procedure was 123 days (range 17 days to 10 months).

Sensitivity, specificity, accuracy, PPV and NPV of MRCP and EUS for the detection of CBD stones are described in Table 2.

MRCP was once false-negative and twice false-positive. EUS showed one false-negative and one false-positive result. One false-positive result of MRCP and EUS occurred in the same patient. Both techniques diagnosed a prepapillary stone millimetric in size. EUS was not followed by ERCP, but intraoperative cholangiography was performed during cholecystectomy 5 days later, which was negative. None of the 12 dynamic coronal MR acquisitions could hereby clearly visualise the sphincter complex in a relaxed state.

In the second false-positive result the analysis of the sphincter complex on MRCP was hampered by a periampullary diverticula of $4 \mathrm{~cm}$.

MRCP yielded one false-negative result concerning a patient with a normal size of intra-and extrahepatic bile ducts. The CBD measured $6 \mathrm{~mm}$ on MRCP, but the sphincter complex was not clearly visible (Fig. 4a). EUS correctly revealed two small distal stones measuring $5 \mathrm{~mm}$ each, also without any associated luminal dilatation (Fig. 4b). 


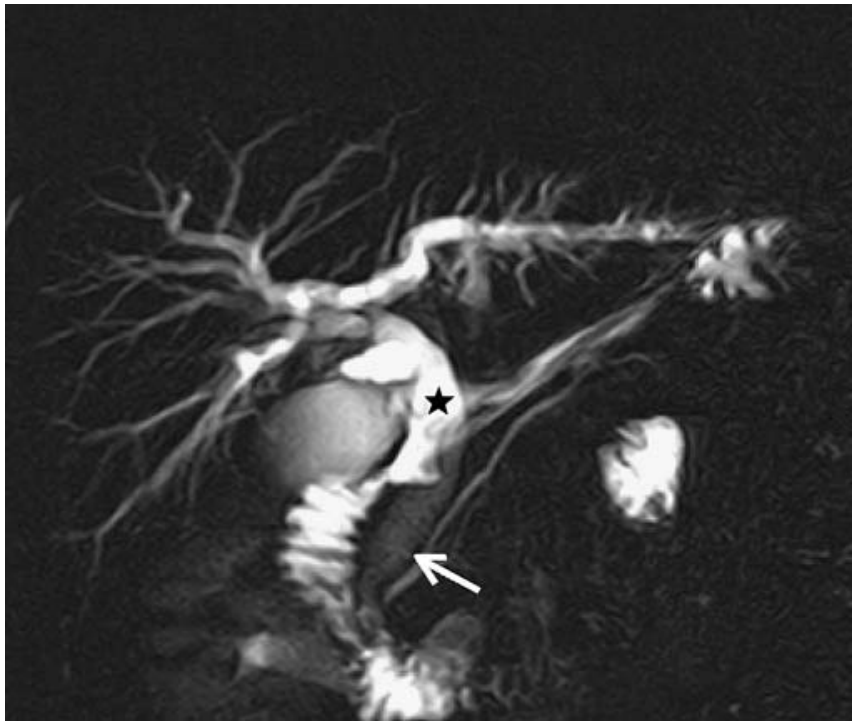

Fig. 3 A 67-year-old woman with acute onset of fever, jaundice and diffuse abdominal pain. Coronal oblique T2-weighted thick-slab $(40 \mathrm{~mm})$ single-shot turbo spin-echo MRCP sequence shows a great amount of biliary sludge accumulating in the lower CBD (arrow) with consecutive dilatation (star), also extending to the intrahepatic bile ducts. These findings were confirmed by EUS and ERCP

EUS was false-negative in one patient with acute pancreatitis and associated cholangitis. MRCP showed at least three distal CBD stones without associated CBD dilatation (Fig. 5). Immediate ERCP including sphincterotomy confirmed the MR result.

Inter-observer agreement between the two radiologists for the detection of CBD stone disease was 0.81 , therefore excellent according to the ratings of Fleiss [31]. Disagreement between the two readers occurred in five cases, among them two patients with CBD stones measuring 2 and $4 \mathrm{~mm}$ in diameter, respectively; therefore nonobstructive (one of them shown in Fig. 5). Both radiologists agreed on the correct diagnosis of 15 patients with choledocholithiasis. They also agreed on the single false-negative result of MRCP (Fig. 4a) and on one of the two false-positive results of MRCP, in which image

Table 2 Statistical results of MRCP and EUS for the detection of choledocholithiasis resulting from our study and in comparison with the previous publications (Sens sensitivity, Spe specificity, Acc analysis was difficult because of a periampullary duodenal diverticula. Concerning the second false-positive result of MRCP, there was disagreement since one of the two radiologists' results had been true negative before consensus reading.

Finally, there were two negative MRI examinations in which one of the two observers made a false-positive diagnosis, among them a 92-year-old and an unco-operative patient presenting with acute pancreatitis of suspected biliary origin. MRCP was technically suboptimal because of many movement artifacts.

In seven out of 18 patients $(38.9 \%)$ with choledocholithiasis, the CBD was of normal size on MRCP, among them four cases with very small calculi measuring $2 \mathrm{~mm}$ in diameter $(n=4)$.

Dilated CBD seen on MRI agreed well with the presence of dilatation diagnosed by EUS ( $k=0.66$ ). Even the degree of CBD dilatation showed a very significant and positive correlation between both imaging modalities (Pearson's correlation coefficient, $r=0.81, P<0.0001$ ).

Taking into account the largest diameter in case of several stones, the stone size measured by EUS and MRI also significantly and positively correlated with each other (Pearson's regression coefficient, $r=0.66, \quad P<0.0015$ ). Stone size varied between 2 and $8 \mathrm{~mm}$ on both imaging modalities. The average stone size was $4.9 \mathrm{~mm}$ on MRCP and $5.5 \mathrm{~mm}$ on EUS.

On dynamic MRCP, the Vaterian sphincter complex was visualised in $26 \%$ of cases at the first repetition $(n=15)$, in $72 \%(n=35)$ at least once during the 12 repetitions, and in $12 \%(n=7)$ not at all. The average visualisation of the sphincter was 3.44 times for the given 12 repetitive images.

\section{Discussion}

There is no international consensus about the exact role of imaging techniques in the diagnosis and management of choledocholithiasis $[21,28]$. In patients with clinical symptoms strongly suggesting choledocholithiasis, such as typical abdominal pain and clearly dilated CBD seen on

accuracy, $P P V$ positive predictive value, $N P V$ negative predictive value. All these figures are given in percentage)

\begin{tabular}{|c|c|c|c|c|c|c|c|c|c|c|c|}
\hline \multirow[t]{2}{*}{ Reference } & \multirow{2}{*}{$\begin{array}{l}\text { Patients included } \\
\text { (with CBD stones) }\end{array}$} & \multicolumn{5}{|c|}{ MRCP } & \multicolumn{5}{|l|}{ EUS } \\
\hline & & Sens & Spe & Acc & PPV & NPV & Sens & Spe & Acc & PPV & NPV \\
\hline 6 & $32(11)$ & 100 & 72.7 & 82.2 & 62.5 & 100 & 100 & 95.4 & 96.9 & 90.9 & 100 \\
\hline 13 & $50(9)$ & 91 & 94 & 92 & 97 & 84 & 97 & 88 & 94 & 94 & 94 \\
\hline 9 & $30(5)$ & 40 & 96 & 86.7 & 66 & 88 & 80 & 95 & 93.3 & 80 & 96 \\
\hline 12 & $47(16)$ & 87.5 & 96.6 & 93.6 & 93.3 & 93.3 & 93.8 & 96.6 & 95.7 & 93.8 & 96.5 \\
\hline 10 & $28(24)$ & 88 & 75 & 86 & 95 & 50 & 100 & 50 & 93 & 92 & 100 \\
\hline Our study & $57(18)$ & 94.8 & 94.4 & 94.7 & 97.4 & 89.5 & 97.4 & 94.4 & 96.5 & 97.4 & 94.4 \\
\hline
\end{tabular}




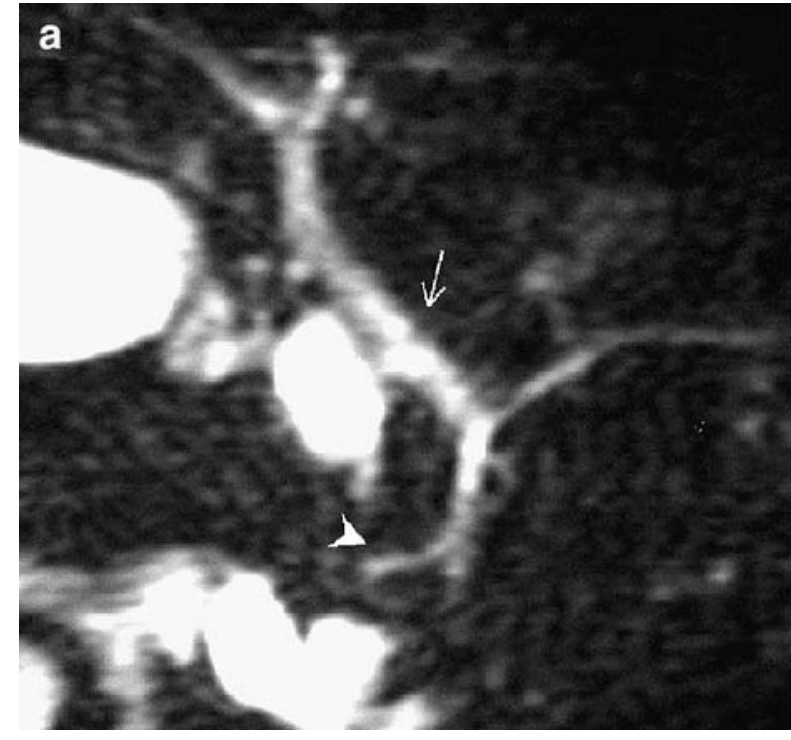

Fig. 4a, b A 91-year-old man presenting in emergency with the clinical picture of cholangitis. a Coronal oblique T2-weighted thickslab single-shot turbo spin-echo MRCP sequence is not of excellent technical quality because of patient's advanced age associated with minor compliance. MR result is false-negative since it does not

transabdominal US associated with jaundice and significantly increased serum bilirubin level, therapeutic ERCP is

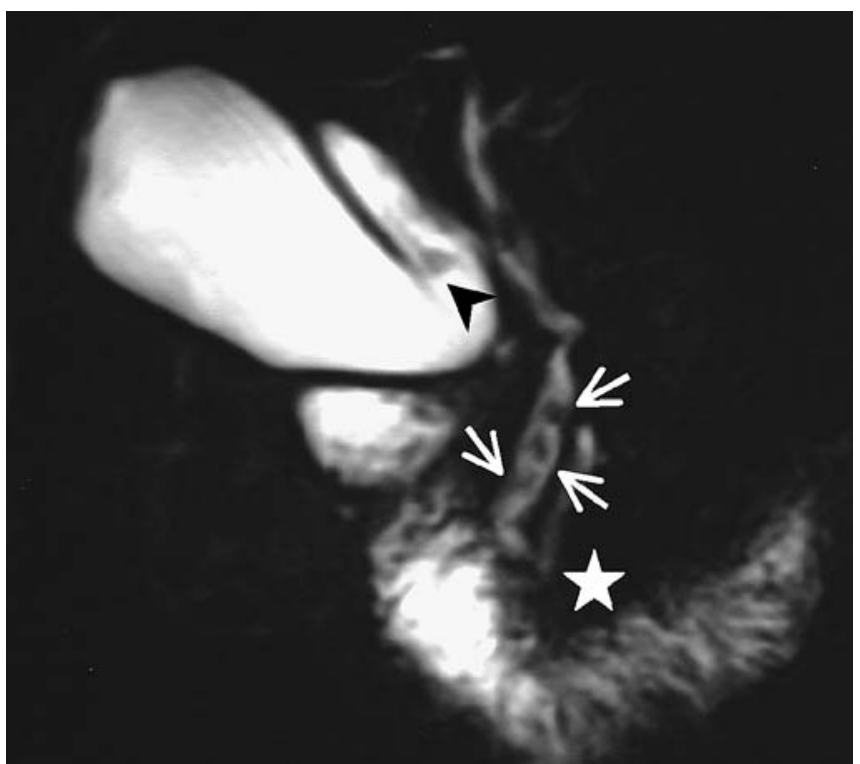

Fig. 5 An 82-year-old woman, known for deafness and senile dementia, with acute pancreatitis of suspected biliary origin and associated cholangitis. Coronal oblique T2-weighted thick-slab single-shot turbo spin-echo MRCP sequence reveals at least three lacunar images (arrows) corresponding to three calculi of the lower portion of CBD. Neither extra-nor intrahepatic bile duct dilatation is seen on MRCP. Note associated gall bladder stone (arrowhead) and the nice visualisation of the sphincter complex at a relaxed state (star). EUS, performed the same day as MRI, did not reveal any of the CBD stones detected by MRI. These, however, were confirmed by immediately following ERCP reveal any signal void neither luminal dilatation of CBD (arrow). Note the anatomical variant of pancreas divisum (arrowhead). b EUS performed the same day reveals two small non-obstructive calculi of the distal CBD measuring $5 \mathrm{~mm}$ each (arrows), extracted by following ERCP

often immediately performed. The typical indication for EUS as well as MRCP is the search of choledocholithiasis in patients with a low and intermediate probability of stone disease in order to avoid purely diagnostic ERCP $[12,14]$. Hence, both techniques become competitive procedures, directly compared by this study relying on dynamic MRI of the extrahepatic bile duct.

EUS has been shown to be of particular advantage for very small stones or sludge even in the non-dilated CBD [14]. According to Scheiman et al. [9] and Buscarini and Buscarini [7], EUS is the most cost-effective initial imaging modality for patients with extrahepatic biliary disease. However, EUS remains a semi-invasive technique, necessitating neuroleptanesthesia, and in more complicated cases even complete anesthesia. An important advantage of EUS over MRCP is the possibility to proceed immediately to ERCP in case of a positive result, the patient remaining on the table still under anaesthesia. Treatment can therefore be performed during the same session, while ERCP has to be organised in a second step after a positive result of MRCP. However, the latter remains completely noninvasive, and therefore better tolerated than endoscopic procedures by the majority of patients [29].

Previous studies focusing on MRCP and choledocholithiasis $[16,20]$ have revealed two diagnostic challenges in particular. The first is to detect small stones, measuring less than $6 \mathrm{~mm}[10,25,30]$, which are not associated with CBD dilatation [18]. The second is to detect prepapillary stones, which may be impacted in the distal CBD. The percentage of small, therefore non-obstructive, calculi is generally under-estimated, representing $38.9 \%(n=7)$ of patients of our study population with proven choledocholithiasis. 
According to Becker et al. [16], tiny calculi, as small as $2 \mathrm{~mm}$ in diameter, without secondary CBD dilatation are best displayed on the axial source images because they are perpendicular to the axis of the CBD. They may be obscured by surrounding hyperintense bile on the MIP or single-shot thick-slice coronal sequence.

The second diagnostic challenge, the detection of prepapillary stones, depends on the individual MRCP technique used. So far, there has not been any published consensus about the optimal MRCP technique for the investigation of choledocholithiasis $[15,28]$. In order to eliminate hyperintense gastric and duodenal content possibly overlapping the bile ducts, the administration of oral contrast and/or patient fasting has been recommended $[12,16,22,31]$. However, in our study we did not do such patient preparation. Instead, we relied on the presence of a sufficient amount of fluid within the duodenal lumen allowing for the exact localization of the papilla of Vater [26].

Most authors first perform axial slices of a fast or turbo spin-echo T2-weighted MR sequence, followed by projecting imaging, which is heavily T2-weighted single-shot fast or turbo spin echo sequences in the coronal oblique plane. The latter are best performed as thick slabs of 20 - to $50-\mathrm{mm}$ thickness $[6,12,13,15-17,31]$. To our knowledge, the use of dynamic or repetitive thick-slab MR sequences in this regard has not yet been reported.

Obviously, for reliable detection or exclusion of any CBD stone visualisation of the entire length of the lumen is mandatory. The distal part of the CBD, including the Vaterian sphincter complex, is the most difficult anatomical structure to demonstrate by MRCP, because of its periodic contractile activity (i.e. 3-4 contractions/min per 4-5 s) [32] (Fig. 6), and due to the small size of the intrasphincteric portion of the duct [30]. Therefore, in at least $15-20 \%$ of MRCP examinations the luminal delineation is not achieved [26]. Since MRI is performed in the physiologically non-distended state of the biliary system, its incomplete demonstration at MRCP does not necessarily indicate disease [15]. However, it can simulate or dissimulate $\mathrm{CBD}$ stones. False-negative readings of MRCP can rarely occur, because small or impacted calculi at the distal CBD or ampulla are very difficult, or even impossible to detect, whenever the sphincter is contracted $[16,22,26]$. False-positive readings of MRPC are more frequent and mostly due to a forcefully contracted sphincter with retrograde invagination appearing as so-called "pseudo-calculus sign", [33] or after stone migration causing edema of the papilla of Vater $[16,36]$. In the study of Sugiyama et al. [25], comparing MRCP with US and ERCP, 22 (64.7\%) out of 34 patients with choledocholithiasis presented with calculi situated in the distal part of the CBD. This stresses the importance to visualise the entire length of the CBD in order to achieve a confident diagnosis.

According to Dalal et al. [35], the intravenous injection of the sphincter-relaxing agent glucagon during MRCP is able to improve the visualisation of the CBD and ampulla of Vater. However, these authors did not use any control group for their evaluation, in contrast to van Hoe et al. [26], who could not prove any apparent effect on the visibility of the most distal portion of the CBD secondary to intravenously injected glucagon. The easiest and possibly only way to reliably visualise the sphincter is, therefore, to consecutively perform several repetitive MRCP sequences, always centred on the sphincter complex during successive episodes of breath-holding. The chosen section thickness must be a compromise between spatial resolution, which increases with thinner slices, and the chances of constant visualisation of the sphincter complex, which increases
Fig. 6a, b A 93-year-old woman known for gallbladder stones (star), at present complicated by biological cholestasis (elevated bilirubin levels). a Coronal oblique T2-weighted thick-slab single-shot turbo spinecho MRCP sequence demonstrates normal size $(7 \mathrm{~mm})$ of the $\mathrm{CBD}$, but the distal portion of CBD cannot be analysed, since the sphincter is contracted. b Due to several repetitions of the same coronal oblique T2weighted thick-slab $(40 \mathrm{~mm})$ single-shot turbo spin-echo MRCP sequence the whole length of CBD becomes visible and the sphincter complex is nicely seen at a relaxed state (arrow), therefore prepapillary stone can reliably be excluded
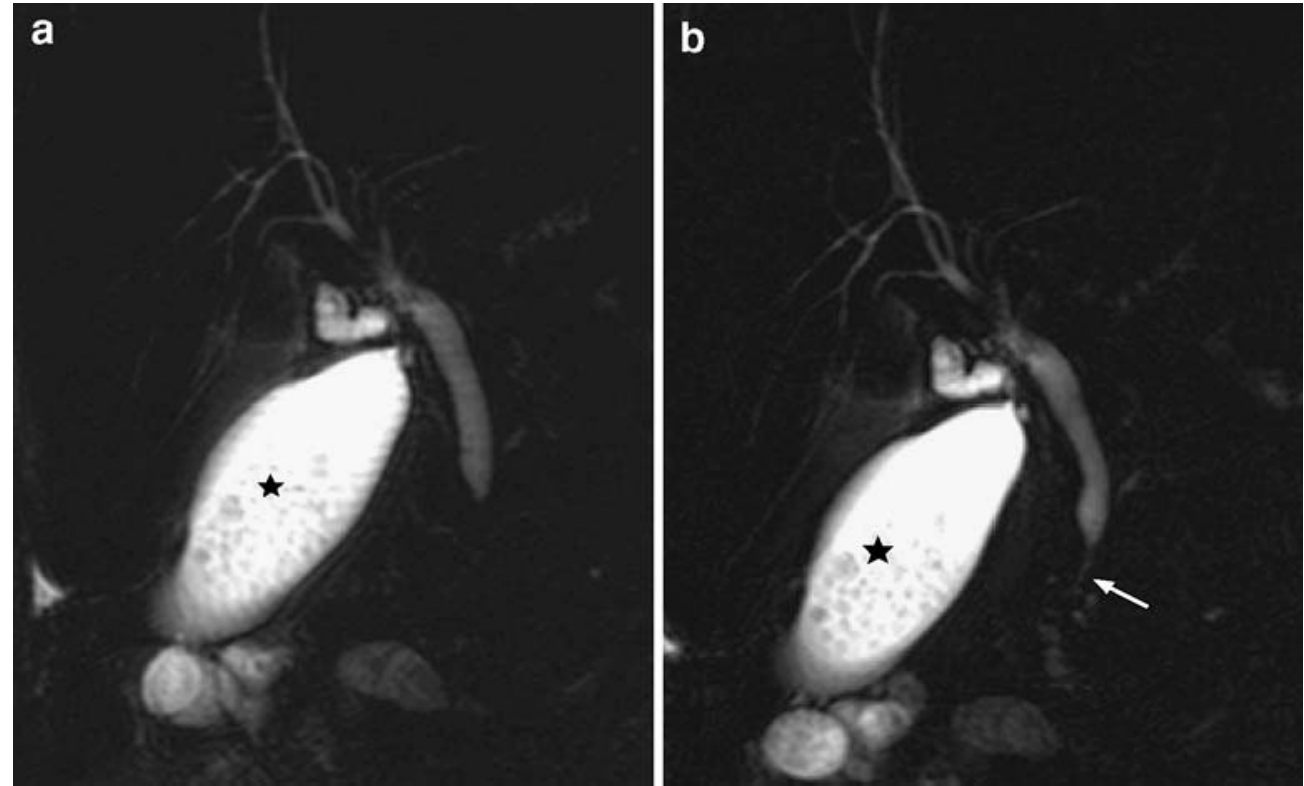
with slice thickness [26]. Furthermore, there is a large interindividual variability for the exact anatomic localization of the papilla of Vater. The number of image repetitions necessary to obtain an adequate visualisation of the sphincter complex in a relaxed state therefore greatly differs among the patients, as it did in our study.

Thanks to the 12 repetitions of the dynamic coronal 40$\mathrm{mm}$ thick-slab single-shot projection MRCP centred on the sphincter complex, the entire CBD had become completely visible in $72 \%(n=35)$ of our patients, while it was seen in only $26 \%(n=15)$ during the first repetition, which means an increase of $46 \%(n=20)$. This improved visualisation of the distal CBD enabled us to confidently exclude the presence of any calculus within the CBD, especially in these 20 patients in whom the acquisition of one single projection MRCP would have caused a false-positive result because of sphincter contraction.

In seven patients of our study, the sphincter complex was not visible at all, despite the 12 repetitions performed. We therefore had to rely on axial sequences for the evaluation of the distal CBD, still keeping in mind that MRCP altogether remains suboptimal in diagnostic quality. In these seven patients, the sphincter complex might have been seen if we had acquired even more repetitive images. Van Hoe et al. [26] described a high inter-indivual variability in this regard, ranging from 2 to 18 slices, even in the absence of sphincter dysfunction. However, our choice to perform exactly 12 repetitions resulted from a practical compromise: to get enough sequential slices necessary to achieve a confident diagnosis and to respect a length of MR examination time still well-accepted by our patients.

To our knowledge, there have been five previous publications directly comparing EUS and MRCP, among them three $[6,10,12]$ exclusively dealing with choledocholithiasis $[6,9,10,12,13]$ (Table 2). Similar to our study, most of the diagnostic results of EUS are slightly better than these of MRCP. However, the differences are mostly not statistically significant. None of these authors used dynamic MR sequences centred on the sphincter complex. They all performed heavily T2-weighted single-shot projection MRCP in the coronal or coronal oblique plane, mostly acquiring thick slices of $20 \mathrm{~mm}$, with the exception of Scheiman and co-workers, who used thin slices of $5 \mathrm{~mm}$, later reconstructed as MIP. The latter are considered of suboptimal quality and less useful for detection of small stones especially, when compared with thick slabs $[17,26$, 34]. This partly explains the poor sensitivity of MRCP reported by these authors [9]. The small size of stones (3$6 \mathrm{~mm}$, average size $=4 \mathrm{~mm}$ ) in this study may also play a role. Statistical interpretation is also difficult because of the low prevalence of choledocholithiasis and the small number of patients $(n=5)$ with a positive result [9].

MR image analysis of the five publications cited above was exclusively done by one radiologist $[6,9]$ or by two in consensus [12, 13], but none of them studied the reproducibility of the MR results obtained by several and independent readings. Our aim was to stress the objective nature of image interpretation by calculating the interobserver agreement between two observers. The excellent kappa value we obtained $(\mathrm{K}=0.8)$ is consistent with the results of previous studies in this regard, where MRCP was mostly compared with ERCP [16, 18, 19, 22, 24].

Our good agreement $(\kappa=0.66)$ between EUS and MRCP concerning CBD dilatation has been confirmed by the study of Aubé et al. [12], who obtained a kappa value slightly superior than ours $(K=0.86)$. The highly significant correlation of stone size between both modalities resulting from our study agrees with the results of de Lédinghen et al. [6]. Our five disconcordant readings were due to suboptimal technique (movement artefacts), anatomic variants (periampullary duodenal diverticula) and presence of small, non-obstructive CBD stones.

Dynamic bile duct imaging has recently been described by drip infusion cholangiography using multidetector CT (MDCTCh) [36, 37]. Compared with MRCP, MDCTCh showed slightly better diagnostic values in 15 patients with choledocholithiasis (MRCP: sensitivity 80\%, specificity $88 \%$, MDCTCh: sensitivity $87 \%$, specificity $96 \%$ ). However, the technique of MRCP only consisted of MIP images without any dynamic imaging, and the delay between MDCTCh and MRCP was 2 weeks, therefore quite long [36]. Finally, MDCTCh is an ionizing procedure and necessitates parenteral contrast injection, which has some limitations, such as serum bilirubin levels and adverse reactions.

Our study shows several limitations. First, the median time interval between MRCP, respectively EUS and ERCP or intraoperative cholangiography was 4.1 days. This delay may be responsible for the one false-positive result we met with MRCP and EUS. Both diagnosed a prepapillary millimetric-sized stone in one patient, while intraoperative cholangiography performed 5 days later was normal. Meanwhile, spontaneous migration may have occurred. Our time interval lies, however, within the limits reported in the literature, ranging between $5 \mathrm{~h}$ and 2 weeks [10, 12, $13,21]$.

Secondly, we cannot exclude the possibility that one patient of our study population presented a possible sphincter dysfunction. In general, we consider 12 repetitive images of MRCP as sufficient for the visualisation of the sphincter complex at least once in a relaxed state. Nevertheless, this failed in $12 \%(n=7)$ of our patients, mostly due to inconstant breathing or reduced patient collaboration because of the severity of the disease. Another explanation may be the limited spatial and contrast resolution of our hardware equipment, the 1-T magnet. However, we think, that our study still reflects current technical equipment present in radiological departments for routine clinical settings worldwide.

Finally, our gold standard for choledocholithiasis was ERCP, although its accuracy is not $100 \%[16,22,28]$. 
ERCP remains an operator-dependent technique. In the hands of experienced biliary endoscopists, $94-100 \%$ of CBD stones may be detected if the bile duct is successfully opacified [38]. Very small stones, in particular, suspected on EUS or MRCP, may have not been visualised or may have migrated beforehand. This should be kept in mind for any study dealing with ERCP and biliary calculi.

In conclusion, our prospective comparison of MRCP and EUS for the detection of choledocholithiasis yielded statistically similar diagnostic values for both techniques. Therefore, as a completely non-invasive procedure, MRCP should be preferred to select patients requiring therapeutic ERCP. We stress the importance of repetitive thick-slab MRCP sequences centred on the sphincter complex achieving its visualisation in a relaxed state, in order to overcome the well-known diagnostic drawbacks inherent in the MRCP technique. Achieving thus a higher diagnostic value than without dynamic MR imaging, we think that sequential thick-slab MRCP acquiring the same coronal slice at least 12 times should routinely be applied for the detection of choledocholithiasis. A confident diagnosis made on MRCP requires visualisation of the entire length of the CBD.

In MRCP-negative patients and persistent clinical concern for choledocholithiasis it seems appropriate to proceed to EUS for the evaluation of microlithiasis, with the possibility of therapeutic ERCP during the same session.

\section{References}

1. Hermann RE (1989) The spectrum of biliary stone disease. Am J Surg 158:171-173

2. Cohen SA, Siegel JH, Kasmin FE (1996) Complications of diagnostic and therapeutic ERCP. Abdominal Imaging 21:385-394

3. Loperfido S, Angelini G, Benedetti G, Chilovi F, Costan F, de Berardinis F, de Bernardin M, Ederle A, Fina P, Fratton A (1998) Major early complications from diagnostic and therapeutic ERCP: a prospective multicenter study. Gastroint Endosc 48:1-10

4. Rieger R, Wayand W (1995) Yield of prospective, non-invasive evaluation of the common bile duct combined with selective ERCP/sphincterotomy in 1930 consecutive laparoscopic cholecystectomy patients. Gastrointest Endosc 42:6-12

5. Palazzo L, Girollet PP, Salmeron M et al (1995) Value of endoscopic ultrasonography in the diagnosis of common bile duct stones: comparison with surgical exploration and ERCP. Gastrointest Endoscop 42:225-231

6. De Lédinghen V, Lecesne R, Raymon J-M et al (1999) Diagnosis of choledocholithiasis: EUS or magnetic resonance cholangiography? A prospective controlled study. Gastroint Endosc 49:26-31

7. Buscarini E, Buscarini L (1999) The role of endosonography in the diagnosis of choledocholithiasis. Eur J Ultrasound 10:117-125
8. Amouyal P, Amouyal G, Levy P, Tuzet S, Palazzo L, Vilgrain V, Gayet B, Belghiti J, Fekete F, Bernades P (1994) Diagnosis of choledocholithiasis by endoscopic ultrasonography. Gastroenterology 106:1062-1067

9. Scheiman JM, Ruth MM, Barnett JL, Elta GH, Nostrant TT, Chey WD, Francis IR, Nandi PS (2001) Can endoscopic ultrasound or magnetic resonance cholangiopancreatography replace ERCP in patients with suspected biliary disease? Am J Gastroenterol 96:2900-2904

10. Kondo $\mathrm{S}$, Isayam $\mathrm{H}$, Akahane $\mathrm{M}$ et al (2005) Detection of common bile duct stones: comparison between endoscopic ultrasonography, magnetic resonance cholangiography, and helical-computed-tomographic cholangiography. Eur J Radiol 271-275

11. Prat F, Amouyal G, Amouyal P et al (1996) Prospective controlled study study of endoscopic ultrasonography and endoscopic retrograde cholangiography in patients with suspected common biled duct lithiasis. Lancet 347:75-79

12. Aubé C, Delorme B, Yzet T, Burtin P, Lebigot J, Pessaux P, Gondry-Jouet C, Boyer J, Caron C (2005) MR cholangiopancreatography versus endoscopic sonography in suspected common bile duct lithiasis: a prospective, comparative study. AJR Am J Roentgenol 184:55-62

13. Materne R, Van Beers BE, Gigot JF, Jamart J, Geubel A, Pringot J, Deprez P (2000) Extrahepatic biliary obstruction: magnetic resonance imaging compared with endoscopic ultrasonography. Endoscopy 32:3-9

14. Deprez P (2000) Approach of suspected common bile duct stones: endoscopic ultrasonography. Acta Gastroenterol Belg 63:295-298
15. Vitellas KM, Keogan MT, Spritzer CE, Nelson RD (2000) MR cholangiopancreatography of bile and pancreatic duct abnormalities with emphasis on the single-shot fast spin-echo technique. RadioGraphics 20:939-957

16. Becker CD, Grossholz M, Becker M, Mentha G, de Peyer R, Terrier F (1997) Choledocholithiasis and bile duct stenosis: diagnostic accuracy of MR cholangiopancreatography. Radiology 205:523-530

17. Ernst O, Calvo M, Serent G, Mizrahi D, Carpentier F (1997) Breath-hold MR cholangiopancreatography using a HASTE sequence: comparison of single-slice and multislice acquisition techniques. AJR Am J Roentgenol 169:1304-1306

18. Guibaud L, Bret PM, Reinhold C, Atri M, Barkun AN (1995) Bile duct obstruction and choledocholithiasis: diagnosis with MR cholangiography. Radiology 197:109-115

19. Soto JA, Barish MA, Alvarez O, Medina S (2000) Detection of choledocholithiasis with MR cholangiography: comparison of three-dimensional fast spin-echo and single-and multisection half-fourier rapid acquisition with relaxation enhancement sequences. Radiology 215:737-745

20. Varghese JC, Liddell RP, Farrell MA, Murray FE, Osborne DH, Lee MJ (2000) Diagnostic accuracy of magnetic resonance cholangiopancreatography and ultrasound compared with direct cholangiography in the detection of choldocholithiasis. Clin Radiol 55:25-35 
21. Pamos S, Benage A, Médina E, Martinez Sanjuan V (2003) Prospective evaluation of magnetic resonance cholangiopancreatography in patients with biliary disease: comparative study with conventional ultrasonography and endoscopic retrograde cholangiopancreatography. Dig Liv Dis 35:186-192

22. Reinhold C, Taourel P, Bret PM, Cortas GA, Mehta SN, Barkun AN, Wang L, Tafazoli F (1998) Choledocholithiasis: Evaluation of MR cholangiography for diagnosis. Radiology 209:435-442

23. Taylor ACF, Little AF, Hennessy OF, Banting SW, Smith PJ, Desmond PV (2002) Prospective assessment of magnetic resonance cholangiopancreatography for noninvasive imaging of the biliary tree. Gastrointest Endosc 55:17-22

24. Boraschi P, Neri E, Braccini G et al (1999) Choledocholithiasis: Diagnostic accuracy of MR cholangiopancreatography. Three-year experience. Mag Res Imag 17:1245-1253

25. Sugiyama M, Atomi Y, Hachiay J (1998) Magnetic resonance cholangiography using half-fourier acquisition for diagnosing choledocholithiasis. Am J Gastroenterol 93:1886-1890
26. van Hoe L, Gryspeerdt S, Vanbeckevoort D, De Jaegere T, Ban Steenbergen W, Dewandel P, Baert AL, Marchal G (1998) Normal Vaterian sphincter complex: Evaluation of morphology and contractility with dynamic single-shot MR cholangiopancreatography. AJR Am J Roentgenol 170:1497-1500

27. Fleiss JL (1985) Statistical methods for rates and proportions, 2nd edn. Whiley, New York, pp 211-236

28. Bret PM, Reinhold C (1997) Magnetic resonance cholangiopancreatography. Endoscopy 29:472-486

29. Menon K, Barkun AN, Romagnuolo J, Friedman G, Mehta SN, Reinhold C, Bret PM (2001) Patient satisfaction after MRCP and ERCP. Am J Gastroenterol 96:2646-2650

30. Zidi SH, Prat F, Le Guen O, Rondeau Y, Rocher L, Fritsch J, Choury AD, Pelletier G (1999) Use of magnetic resonance cholangiography in the diagnosis of choledocholithiasis: prospective comparison with a reference imaging method. GUT 44:118-122

31. Coppens E, Metens T, Winan C, Matos C (2005) Pineapple juice labeled with gadolinium: a convenient oral contrast for magnetic resonance cholangiopancreatography. Eur Radiol 15:2122-2129

32. Staritz M (1988) Pharmacology of the sphincter of Oddi. Endoscopy 20:171-174
33. van Hoe L, Mermuys K, Vanhoenacker P (2004) MRCP pitfalls. Abdom Imaging 29:360-387

34. Régent D (1998) La cholangiopancréatiographie IRM et l'exploration des structures canalaires de demain: tournedos ou carpaccio? J Radiol 79:107-111

35. Dalal PU, Howlett DC, Sallomi DF, Marchbank ND, Watson GMT, Marr A, Dunk AA, Smith AD (2004) Does intravenous glucagon improve common bile duct visualisation during magnetic resonance cholangiopancreatography? Results in 42 patients. Eur J Radiol 49:258-261

36. Okada M, Fukada J, Toya K, Ito R, Ohashi T, Yorozu A (2005) The value of drip infusion cholangiography using multidetector-row helical CT in patients with choledocholithiasis. Eur Radiol 15:2140-2145

37. Eracleous E, Genagritis M, Papanikolaou N, Kontou AM, Prassopoullos P, Chrysikopoulos H, Allan P, Gourtsoyiannis N (2005) Complementary role of helical CT cholangiography to MR cholangiography in the evaluation of biliary function and kinetics. Eur Radiol 15:2130-2139

38. Lo KS, Chen J (1996) The role of ERCP in choledocholithiasis. Abdom Imaging 21:120-132 